\title{
«Hilfe aus dem App-Store?»: Eine systematische Übersichtsarbeit und Evaluation von Apps zur Anwendung bei Depressionen
}

\author{
Yannik Terhorst $^{\mathrm{a}} \quad$ Eva-Maria Rathner $^{\mathrm{a}}$ Harald Baumeister $^{\mathrm{a}}$ Lasse Sander $^{\mathrm{b}}$ \\ ${ }^{a}$ Abteilung für Klinische Psychologie und Psychotherapie, Institut für Psychologie und Pädagogik, Universität Ulm, Ulm, Deutschland; \\ ${ }^{b}$ Abteilung für Rehabilitationspsychologie und Psychotherapie, Institut für Psychologie, Universität Freiburg, Freiburg i.Br., Deutschland
}

\section{Schlüsselwörter}

Systematische Übersichtsarbeit · Depression · Apps .

Mobile Health

\section{Zusammenfassung}

Hintergrund: In Deutschland sind etwa 4,9 Millionen Menschen an Depressionen erkrankt. Depressionen sind für die Betroffenen und die Gesellschaft mit enormen Belastungen verbunden. Gesundheits-Apps haben hier das Potenzial, die Versorgungslage zu verbessern. Das Ziel dieser systematischen Übersichtsarbeit ist es, die Qualität, Inhalte und Praxisrelevanz von deutschsprachigen Apps für die Anwendung bei Depressionen zu untersuchen. Methode: Die deutschen Google-Play- und iTunes-Stores wurden systematisch nach Apps durchsucht, die explizit mit der Thematik "Depression/Depressivität» warben. Die so ermittelten Apps wurden mithilfe einer Skala zur Einschätzung der Qualität (Mobile Application Rating Scale) von 2 unabhängigen Gutachtern bewertet. Apps mit überdurchschnittlichem Rating wurden von 2 praktisch tätigen Verhaltenstherapeuten im Hinblick auf ihren Nutzen für die klinische Praxis beurteilt. Ergebnisse: Von 1156 identifizierten Apps wurden 38 eingeschlossen. Inhaltlich reichten diese von Informations- bis zu Interventions-Apps. Die Apps wiesen eine mittlere Gesamtqualität auf $(M=3,01$; Standardabweichung $=0,56)$. Vier Apps zeigten überdurchschnittliche Werte. Sie wurden durch 2 Psychotherapeuten getestet und als bedingt empfehlenswert für die klinische Praxis beurteilt. $\mathrm{Zu}$ keiner der eingeschlossenen Apps konnte eine Wirksamkeitsstudie gefunden werden. Schlussfolgerungen: Deutschsprachige Depressions-Apps weisen qualitative Mängel auf. Zusätzlich fehlt es an klinischen Studien zum Nutzen und zu Risiken, weshalb der Einsatz in der klinischen Praxis nur bedingt empfohlen werden kann. Ein Gütesiegel für qualitativ hochwertige und praxisrelevante Gesundheits-Apps könnte Nutzer vor Fehlinformationen und Missbrauch schützen und Leistungserbringern den Einsatz digitaler Medien substanziell erleichtern.

(C) 2018 S. Karger GmbH, Freiburg

Zur besseren Lesbarkeit wird in diesem Artikel das generische Maskulinum verwendet. Gemeint sind jedoch alle Geschlechter.

\author{
Keywords \\ Systematic Review · Depression · Apps · Mobile Health
}

\section{Summary \\ 'Help from the App Store?': A Systematic Review of \\ Depression Apps in German App Stores}

Background: Approximately 4.9 million individuals in Germany are affected by depression. Depression causes high personal and social burdens. Apps may improve health care in this field. In this systematic review, the quality, content, and practical relevance of German-language depression apps are assessed. Methods: A systematic search for apps explicitly targeting depression was conducted in the German app stores of Google Play and iTunes. The quality of the identified apps was rated by 2 independent researchers by means of a scale for the quality assessment of apps (Mobile Application Rating Scale). Apps that were found to be of high quality were evaluated by 2 practicing psychotherapists regarding their practical relevance. Results: 38 out of 1,156 identified depression apps were included in the study. Their content ranged from basic information to specific interventions. The overall quality was average $(\mathrm{M}=$ 3.01 , standard deviation $=0.56$ ). Four high-quality apps were identified. They were evaluated by 2 psychotherapists and recommended with reservations for practical use. No effectiveness study was found for any app included. Conclusions: Depression apps available in German showed an average quality. There is a general lack of evidence. The identified apps can only be recommended with reservations. A quality seal could help by marking high-quality apps with practically relevant contents. Thereby, users could be protected from misinformation and abuse, and therapists would be able to facilitate the use of digital media more easily.

\section{KARGER \\ Fax +497614520714

() 2018 S. Karger GmbH, Freiburg
Yannik Terhorst

Abteilung Klinische Psychologie und Psychotherapie, Institut für Psychologie und Pädagogik, Fakultät Ingenieurwissenschaften, Informatik und Psychologie

Universität Ulm

Albert-Einstein-Allee 47, 89081 Ulm, Deutschland

yannik.terhorst@uni-ulm.de 


\section{Einleitung}

Nach Schätzungen der World Health Organization (WHO) werden Depressionen bis zum Jahr 2030 die weltweit größte Ursache für Krankheitsbelastung sein [WHO, 2011]. Die globale Punktprävalenz für Depression liegt derzeit bei etwa 4,7\%, die Inzidenz bei 3\% [Ferrari et al., 2013b]. Depressionen sind sowohl für die Betroffenen als auch für die Gesellschaft mit hohen Belastungen verbunden [Ferrari et al., 2013a; Sobocki et al., 2006]. In Deutschland sind jährlich etwa 4,9 Millionen Menschen von der Erkrankung betroffen [Jacobi et al., 2014]. Die nationale Versorgungslage ist nicht hinreichend: Bei der Hälfte aller behandlungsrelevanten Depressionsfälle erfolgt keine oder keine ausreichend lange Behandlung [Melchior et al., 2014; Mack et al., 2014]. Von den schweren Fällen bleiben etwa $18 \%$ und von den chronischen $31 \%$ ohne Behandlung [Melchior et al., 2014].

Gesundheits-Apps haben das Potenzial, die aktuelle Versorgungslage zu verbessern, und können sowohl in der Prävention, der Diagnostik und der Therapie eingesetzt werden [Albrecht, 2016a]. Apps könnten unter anderem zu Kosteneinsparungen führen, den Zugang zu evidenzbasierten Angeboten fördern [Endl et al., 2015; Aitken, 2015; Kuhn und Amelung, 2016], die Adhärenz steigern [Aitken, 2015; Kuhn und Amelung, 2016], die Datenverfügbarkeit verbessern [Charani et al., 2014] und zu einer stärken Patientenzentrierung, Patientenautonomie und Selbstbestimmung führen [Kuhn und Amelung, 2016; Nasi et al., 2015; Klasnja und Pratt, 2012; Dicianno et al., 2015; Pejovic et al., 2015; Gupta et al., 2013]. Auch bieten Apps die Chance, eine Anschlussbehandlung, Nachsorge oder Step-Down-Behandlung zu ermöglichen und zu verbessern sowie Betroffenen, die eine therapeutische Behandlung entweder ablehnen oder mit langen Wartezeiten konfrontiert sind, niederschwellig Hilfestellung anzubieten [Price et al., 2014; Andrade et al., 2014]. Zusätzlich erlauben Apps ein alltagsnahes Symptom- und Aktivitäten-Monitoring, steigern die Flexibilität von Behandlungen und verbessern die Behandlungsadhärenz durch Feedback und Erinnerungsfunktionen [Harrison et al., 2011; Whittaker et al., 2012; Carter et al., 2013; Proudfoot und Nicholas, 2010; Warmerdam et al., 2012]. Weiterhin könnten Gesundheits-Apps besondere Zielgruppen erreichen, die durch klassische Versorgungsangebote derzeit nicht erreicht werden [Kuhn und Amelung, 2016].

Die primäre Zugangsvoraussetzung für Gesundheits-Apps ist der Besitz eines Smartphones oder sonstigen geeigneten Endgeräts [Kuhn und Amelung, 2016]. Allerdings ist die Verfügbarkeit solcher Geräte größtenteils flächendeckend gegeben [Google, 2012; Meeker und Liang, 2013; Liu, 2015; Cerwall et al., 2015]. Der weltweite Nutzungsanteil von Smartphones stieg von 2015 bis 2016 um $12 \%$ auf $45,2 \%$ an und liegt in Deutschland bei etwa 71,7\% [Liu, 2015]. Auch die Bereitschaft für den Gebrauch von Apps zum Thema «psychische Gesundheit» ist vorhanden. In einer australischen Umfrage gaben 76\% der Befragten an, dass sie ihr Smartphone für Selbstmanagement und Selbst-Monitoring in Bezug auf ihre psychische Gesundheit nutzen würden, wenn dieses Angebot kostenfrei wäre [Proudfoot et al., 2010]. Eine aktuelle Befragung in
Deutschland zeigte zudem, dass Gesundheits-Apps (Medizin-, Gesundheits-, therapieunterstützende Apps) bereits häufig genutzt wurden [Tomorrow Focus Media, 2015].

Mehrere Studien belegen auch die Effektivität von GesundheitsApps. Im Bereich der Prävention konnten Gesundheits-Apps z.B. das Gesundheitsbewusstsein, die körperliche Fitness und den Gewichtsverlust positiv beeinflussen [Rutz et al., 2016a]. Im Bereich der Therapie gibt es hingegen kaum belastbare Evidenz zum Einsatz von Apps [Rutz et al., 2016b]. Im Bereich der Depression gibt es wenige Befunde und die, die existieren, sind zum Teil uneindeutig [Torous und Powell, 2015; Donker et al., 2013]: In einer systematischen Übersichtsarbeit ermittelten Donker et al. [2013] 4 Studien zur Effektivität von Apps bei Depression. Während in 2 dieser 4 randomisierten kontrollierten Studien Effektstärken von d $=1,95$ bzw. $\mathrm{d}=1,56$ in Bezug auf den Depressionsschweregrad (PHQ-9) zwischen einer Interventionsgruppe mit App und einer AttentionControl-Gruppe ohne App festgestellt wurden [Burns et al., 2011], konnten in den anderen 2 Studien keine Effekte gezeigten werden [Kauer et al., 2012; Reid et al., 2011]. Nichtsdestotrotz werden Therapie-Apps für Patienten und Leistungserbringer (z.B. Ärzte) angeboten [Rutz et al., 2016b]. Auch gibt es Apps für Diagnosezwecke, wobei hier ebenfalls wenige qualitativ hochwertige Studien vorliegen und derzeit keine Aussage über ihren Nutzen getroffen werden kann [Rutz et al., 2016b].

Der Gebrauch von therapie- und gesundheitsbezogenen Apps kann von Risiken und Nebenwirkungen begleitet sein. Ein unzureichender Schutz der Daten und der Privatsphäre oder Behandlungen ohne informierte Einwilligung sind dabei zentrale Risiken [Prentice und Dobson, 2014; Luxton et al., 2011; Albrecht, 2016b]. Ebenso ist das Reaktionsvermögen in akuten Krisen eingeschränkt, und potenzielle Schadensrisiken sind für den Patienten nicht einschätzbar [Luxton et al., 2011]. Obwohl sich derzeit keine Berichte zu konkreten Schäden durch Apps finden lassen, besteht ein generelles Missbrauchs- und Gefährdungspotenzial [Albrecht, 2016b].

In Untersuchungen wie der CHARISMHA-Studie (CHARISMHA = Chances and Risks of Mobile Health Apps) [Albrecht, 2016a] wurden aktuelle Rahmenbedingungen für den Einsatz von Gesundheits-Apps beschrieben und deren Chancen und Risiken umfassend diskutiert. Welche Qualität das aktuelle GesundheitsApp-Angebot in Deutschland hat, ist jedoch unklar. Nutzerratings sind irreführend, da sie von der Benutzerfreundlichkeit und der Funktionalität stärker beeinflusst werden als von der inhaltlichen Qualität [Armstrong, 2015; Bardus et al., 2016; Nicholas et al., 2015]. Neben den Betroffenen haben auch Leistungserbringer Schwierigkeiten, eine ihr Behandlungsangebot ergänzende App zu finden und zu empfehlen [Shen et al., 2015]. Systematische Übersichtsarbeiten sind daher von großer Bedeutung, um die Qualität und die Inhalte von Apps zu überprüfen und potenziellen Nutzern Empfehlungen für den Umgang mit ihnen zu geben. Kürzlich entwickelte multidimensionale Messinstrumente wie die Mobile Application Rating Scale (MARS) [Stoyanov et al., 2015] oder das «Enlight»-Tool [Baumel et al., 2017] sind potenziell geeignet für die Einschätzung der Qualität von Apps. Die MARS ermöglicht es, die Qualität von Apps bezüglich ihrer Nutzereinbindung, Funktio- 
nalität, Ästhetik und Informationsqualität verlässlich einzuschätzen und zu vergleichen [Stoyanov et al., 2015; Bakker et al., 2016].

Die vorliegende Studie zielt auf die Untersuchung verfügbarer Apps, die explizit für mit der Thematik «Depression/Depressivität» werben und dementsprechend in den App-Stores verschlagwortet und erhältlich sind. Folgende Forschungsfragen werden beantwortet:

1) Welche Qualität weisen Apps, die explizit für Depression/Depressivität entwickelt und entsprechend verschlagwortet sind, aus deutschen App-Stores in Bezug auf Nutzereinbindung, Funktionalität, Ästhetik und Informationsgehalt auf?

2) Welche theoretischen Hintergründe und damit verbundenen Inhalte weisen die Apps auf?

3) Welche Apps können für die klinische Praxis empfohlen werden?

\section{Methodik}

\section{Suchstrategie und Prozedere}

Der in der Studie verwendete Suchstring wurde entlang der Störung «Depression» entwickelt. Apps, die mit der Thematik «Depression» bzw. «Depressivität» werben und verschlagwortet sind, wurden bei Google Play und iTunes mit den Suchbegriffen «Depression», «depressiv», «Stimmung», «Stimmungsschwankung», «Schwermut», «affektiv» und «Dysthymie» identifiziert. Mit diesen Begriffen wurde jeweils einzeln gesucht, da die Verwendung von logischen Operatoren und eine Trunkierung nicht möglich waren. Die Suche wurde über das Web-Interface von Google Play und die Anwendungsoberfläche des Programms iTunes am 5. bzw. 6. November 2016 durchgeführt. Die Apps aus Google Play wurden auf ein Huawei Media Pad X2 (Modell: GEM-701L) heruntergeladen, die Apps aus iTunes auf ein iPad mini 4 (Modell: MK9N2FD/A).

\section{Ein- und Ausschlusskriterien}

Apps wurden heruntergeladen, wenn durch den Titel, die Beschreibung, die Bilder oder die Nutzerkommentare deutlich wurde, dass a) die App für Depression entwickelt wurde, b) die App in deutscher Sprache erhältlich war, c) die App über den offiziellen Store herunterladbar war und d) kein sonstiger Ausschlussgrund (App-Bundles: mehrere Apps, die zusammen erhältlich waren) vorlag.

Heruntergeladene Apps wurden in die Untersuchung eingeschlossen, wenn ihre Inhalte a) auf Depression ausgelegt und b) deutschsprachig waren, c) die Apps soweit funktionierten, dass eine Bewertung möglich war und d) kein sonstiger Ausschlussgrund (Inkompatibilität mit den Geräten, Entwicklung/Testphase) vorlag.

\section{Datenextraktion, Evaluationskriterien und Instrumente}

Die Daten wurden von 2 unabhängigen Gutachtern (Y.T. und E.-M.R.) mit der MARS [Stoyanov et al., 2015] erfasst und evaluiert. Die MARS besteht aus einer Klassifikationssektion und einem Qualitätsrating und bedarf eines von den Entwicklern kostenfrei zur Verfügung gestellten Online-Trainings ( $w w w$. youtube.com/watch? $v=25 v B w J Q I O c E$ (zuletzt aktualisiert am 23. August 2017)). Zur Qualitätssicherung des Ratings wurde die Interrater-Reliabilität (IRR) zwischen den Gutachtern berechnet. Eine exzellente Intraklassen-Korrelation (ICC) $\geq 0,75$ wird als hinreichend betrachtet [Fleiss, 1999]. Hätte die Übereinstimmung diesen Wert unterschritten, wäre ein dritter Rater (LS) hinzugezogen worden.

Die MARS weist insgesamt eine exzellente interne Konsistenz von $\alpha=0,90$ und eine gute IRR ( 2 -way mixed ICC $=0,79 ; 95 \%$-Konfidenzintervall (95\%-KI) 0,75 bis 0,83 ) auf [Stoyanov et al., 2015]. Die interne Konsistenz der Subskalen liegt ebenfalls sehr hoch $(\alpha=0,80$ bis 0,89 ; Median $(M d n)=0,85)$, und die IRR der Subskalen reicht von ausreichend bis sehr gut (ICC $=0,50$ bis 0,80 ; $\mathrm{Mdn}=$ 0,65) [Stoyanov et al., 2015]. Die Korrelation zwischen in den App-Stores ver- fügbaren Nutzerratings und dem mittleren Gesamtergebnis der MARS lag im Validierungsprozess bei $(\mathrm{r}(15)=0,55 ; \mathrm{p}<0,05)$ [Hides et al., 2014].

\section{Allgemeine Charakteristika}

Die Klassifikationssektion der MARS wurde leicht abgeändert, um folgende Dimensionen zu erfassen: 1) Name der App, 2) Version der App, 3) Plattform (Android, iOS), 4) Kurzbeschreibung, 5) Nutzerrating, 6) Anzahl der Bewertungen, 7) Store-Kategorie, 8) Preis der Upgrade-Version, 9) technische Aspekte (erlaubt das Teilen von Inhalten oder Nachrichten (z.B. über Facebook, Twitter usw.), besitzt eine App-Community, Passwortschutz, benötigt ein Login, sendet Erinnerungen, braucht Internetzugang), 10) adressierte Altersgruppe.

\section{Qualitätsrating der Apps}

Das Qualitätsrating der MARS besteht aus insgesamt 19 Items, die jeweils in ganzzahligen Stufen von 1 (unpassend) bis 5 (exzellent) bewertet werden können. Die Items sind auf 4 Dimensionen aufgeteilt: 1) Nutzereinbindung (5 Items: Unterhaltung, Interesse, Personalisierung, Interaktivität, Zielgruppe), 2) Funktionalität (4 Items: Performanz, Benutzerfreundlichkeit, Navigation, Gestikdesign), 3) Ästhetik (3 Items: Layout, Graphiken, Visuelle Attraktivität), 4) Informationsqualität (7 Items: Genauigkeit der App-Beschreibung, Ziele, Qualität der Informationen, Quantität der Informationen, Qualität visueller Informationen, Vertrauenswürdigkeit, Evidenz). Zur Bewertung wurde sowohl ein Mittelwert für jede Dimension als auch ein Mittelwert über alle Dimensionen hinweg berechnet [Stoyanov et al., 2015; Hides et al., 2014]. Darüber hinaus wurde die Korrelation zwischen dem Nutzerrating, den Mittelwerten der Subdimensionen und dem Mittelwert über alle Dimensionen berechnet. Für alle Berechnungen wurden die Ratings der beiden Gutachter gemittelt.

\section{Inhalte}

Die Inhalte der Apps wurden mithilfe der modifizierten Klassifikationsseite der MARS erfasst. Zwischen folgenden Inhalten wurde unterschieden: Assessment, Informationen/Aufklärung, Monitoring/Tracking und Interventionselementen. Bei «Interventionselementen» wurde zudem (ergänzt) nach Kampling et al. [2014] zwischen folgenden theoretischen Hintergründen unterschieden:

1) kognitive Verhaltenstherapie (KVT), z.B. Problemlösen, Stressmanagement, Rekonstruktion;

2) psychodynamische Psychotherapie (PP), z.B. psychoanalytische Therapie;

3) Verhaltenstherapie (VT), z.B. Aktivitätsplanung, Expositionstherapie;

4) systemische Therapie (ST), z.B. narrative Therapie;

5) KVT der dritten Welle, z.B. achtsamkeitsbasierte Therapie (= mindfullnessbased cogntive therapy; MBCT), Akzeptanz- und Commitment-Therapie (ACT);

6) humanistische Therapien (HAT), z.B. expressive Therapie, Gesprächspsychotherapie (GPT);

7) integrative Therapien (IT), z.B. interpersonelle Psychotherapie (IPT);

8) andere psychologisch orientierte Therapien, z.B. Bibliotherapie;

9) alternativmedizinische Interventionselemente, z.B. Akupressur.

\section{Empfehlungen für die Praxis}

Apps, die einen hohen Wert in der MARS-Dimension «Informationsqualität» (>4) und einen überdurchschnittlichen MARS-Gesamtmittelwert $(>3.5)$ aufwiesen, wurden von 2 approbierten Psychotherapeuten und Wissenschaftlern (L.S., H.B.) auf ihre Praxisrelevanz geprüft. Die Prüfkriterien waren, ob die Apps:

- die Möglichkeit bieten eine depressive Symptomatik zu messen und die Testergebnisse adäquat vermitteln (Assessment);

- das Tracking von Symptomen einer Depression (Stimmung/Traurigkeit, Interessenverlust, Appetit/Gewichtsänderungen, Schlaf, Aktivitäten, Konzentration, Suizidalität) ermöglichen (Monitoring/Tracking Inhalte);

- Methoden zur Behandlung von Depression beinhalten, die hinreichend untersucht wurden, effektiv sind und mithilfe der Apps adäquat umgesetzt werden (Interventionselemente);

- den nationalen Leitlinien für Depression entsprechend über Depression aufklären (Informationen/Aufklärung) [DGPPN et al., 2015]. 
Abb. 1. Flowchart des Einschlussprozesses der Apps.

\section{Ergebnisse}

\section{Überblick}

Die Suche in Google Play und iTunes ergab ohne Duplikate insgesamt 1156 Treffer. Das Screening ergab, dass 815 der Apps (70,5\%) nicht für Depression entwickelt waren. 274 Apps (23,7\%) waren nicht deutschsprachig. Drei Treffer wurden ausgeschlossen, da es sich um App-Bundles handelte, also eine Gruppe von mehreren Apps, die zusammen zu einem vergünstigten Preis erworben werden können. Nach dem Screening wurden 64 Apps heruntergeladen (eine Liste aller heruntergeladenen Apps kann bei den Autoren angefragt werden). Drei der heruntergeladenen Apps waren nicht für Depression. 19 Apps waren nicht deutschsprachig, und 2 Apps funktionierten nicht ausreichend, um sie zu bewerten. Aus sonstigen Gründen (Inkompatibilität zum Gerät $(n=1)$; in Entwicklung/Testphase $(\mathrm{n}=1))$ wurden 2 Apps ausgeschlossen. Insgesamt gingen 38 Apps in die Analyse ein (Abb. 1).

\section{Allgemeine Charakteristiken}

Von den 38 eingeschlossen Apps waren 27 für das Betriebssystem Android und 11 für iOS entwickelt (eine Liste aller eingeschlossenen Apps kann bei den Autoren angefragt werden). Der

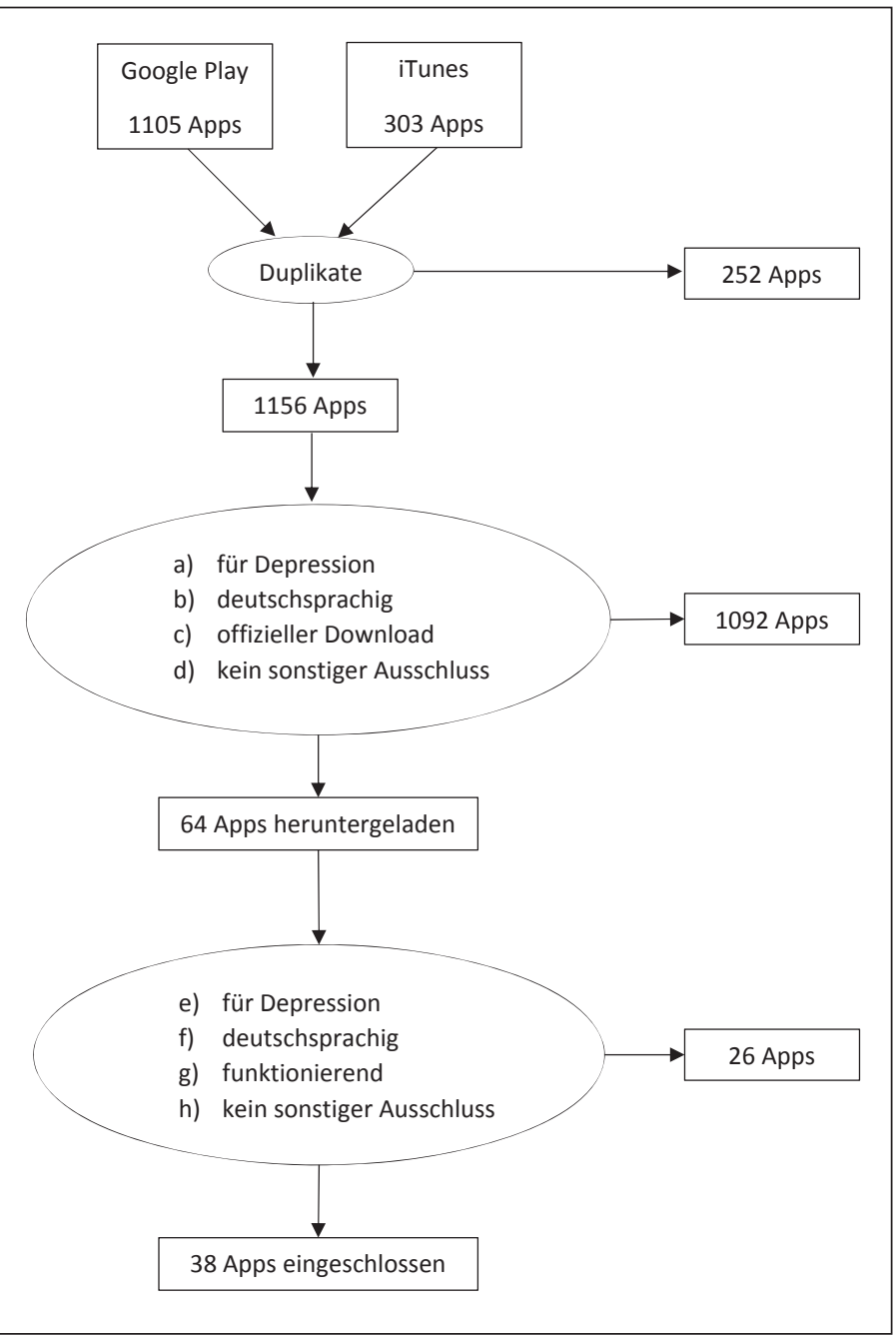

Median des Nutzerratings der Apps lag bei 4,00 ( $M=3,91$; Standardbweichung $(S D)=0,75$ ). Insgesamt waren diese Apps in 7 verschiedenen Kategorien gelistet: «Gesundheit \& Fitness» (n = 19 $(50,0 \%))$, «Medizin» $(n=12(31,6 \%))$, «Lifestyle», «Musik \& Audio» (je $\mathrm{n}=2(5,3 \%))$, «Bücher \& Nachschlagewerke», «Effizienz» und «Unterhaltung» (je $\mathrm{n}=1(2,6 \%)$ ). Die am häufigsten verwendeten Technik-Features waren Teilen-Funktionen ( $\mathrm{n}=14$ $(36,8 \%))$ und Erinnerungsfunktionen $(n=13(34,2 \%))$. Die Erinnerungsfunktionen variierten von simplen Benachrichtigungen bis hin zu Pop-up-Fenstern, die das direkte Erledigen einer anstehenden oder vergessenen Aufgabe ermöglichten. Neun der 38 Apps waren kostenpflichtig. Die Kosten reichten von 0,89 bis 15,95 EUR. Zwei Apps boten einen Passwortschutz an. Keine der Apps spezifizierte in der Beschreibung oder im Programm selbst die Altersgruppe, an die sich ihr Inhalt richtete.

Wissenschaftliche Studien zur Effektivität oder psychometrischen Güte konnten über Google, Google Scholar oder die Entwickler-Website zu keiner der Apps gefunden werden.

\section{Qualitätsrating der Apps}

Die Gesamtqualität der Apps war mit $\mathrm{M}=3,02(\mathrm{SD}=0,51)$ mittelmäßig. In der Subdimension «Funktionalität» lag der Mittelwert 
Tab. 1. MARS-

Rating absteigend nach dem Gesamtmittelwert

\begin{tabular}{|c|c|c|c|c|c|}
\hline Name & $\begin{array}{l}\text { Nutzer- } \\
\text { einbindung }\end{array}$ & Funktionalität & Ästhetik & $\begin{array}{l}\text { Informations- } \\
\text { qualität }\end{array}$ & Gesamt \\
\hline Stimmungstagebuch BETA & 4,2 & 4,5 & 4,67 & 4,17 & 4,38 \\
\hline EnkeApp & 4,2 & 3,75 & 4 & 4 & 3,99 \\
\hline Emotion & 4,2 & 3,75 & 3,33 & 4,33 & 3,90 \\
\hline Back to Job & 3,8 & 3,25 & 3 & 4,17 & 3,55 \\
\hline Depression erleichtern Hypnose & 3,2 & 3,5 & 4 & 2,8 & 3,38 \\
\hline $\begin{array}{l}\text { Akkupressur gegen Depression } \\
\quad \text { (kostenpflichtig) }^{\mathrm{a}}\end{array}$ & 3,2 & 3 & 4 & 3,17 & 3,34 \\
\hline Calmster - innere Ruhe & 3,4 & 3,75 & 3 & 3 & 3,29 \\
\hline Zenify Meditation, Achtsamkeit & 3 & 3 & 4 & 3,17 & 3,29 \\
\hline MoodLog & 3,8 & 3 & 3 & 3,33 & 3,28 \\
\hline Calmster Pro & 3,4 & 2,75 & 4 & 2,83 & 3,25 \\
\hline Depressionstest & 3,4 & 3 & 3 & 3,5 & 3,23 \\
\hline $\begin{array}{l}\text { Get Rid Of Depression With Chinese } \\
\text { Massage Points - FREE Acupressure } \\
\text { Treatment Trainer }\end{array}$ & 3,2 & 3 & 3,33 & 3,33 & 3,22 \\
\hline Bible Promises: auf Deutsch & 3 & 3,75 & 3 & 3 & 3,19 \\
\hline Emotion- Psychologie Test & 3,6 & 3,25 & 3 & 2,83 & 3,17 \\
\hline $\begin{array}{l}\text { Get RidOf Depression With Chinese } \\
\text { Massage Points - PREMIUM } \\
\text { Accupressure Treatment Trainer }{ }^{b}\end{array}$ & 3 & 3 & 3,33 & 3,33 & 3,17 \\
\hline Selbstmanagement Depression & 2,6 & 3,25 & 3 & 3,75 & 3,15 \\
\hline $\begin{array}{l}\text { Depression Test - Am I Depressed Online } \\
\text { Personality Test App }\end{array}$ & 2,8 & 3 & 3 & 3,4 & 3,05 \\
\hline depression-be positive mentality & 3 & 2,75 & 3 & 3,4 & 3,04 \\
\hline WhatsMyM3 & 3 & 3 & 2,67 & 3,5 & 3,04 \\
\hline PHQ-9 Depression Test Questionnaire & 2,8 & 3 & 3 & 3,33 & 3,03 \\
\hline Depressive Verstimmung Scheibe & 2,4 & 3,25 & 3,33 & 3 & 3 \\
\hline $\begin{array}{l}\text { Akkupressur gegen Depression } \\
\quad{\text { (kostenfrei })^{\mathrm{a}}}^{\text {(kosto }}\end{array}$ & 3,2 & 3 & 3 & 2,67 & 2,97 \\
\hline Depressed? & 2,6 & 3 & 3 & 3,2 & 2,95 \\
\hline Lulaj 1 & 2,8 & 3 & 3 & 2,8 & 2,9 \\
\hline Depressions-Test & 2,8 & 2,5 & 2,67 & 3,6 & 2,89 \\
\hline Neu! Depression Test & 2,4 & 2,75 & 3 & 3,2 & 2,84 \\
\hline Beethoven - Klassische Musik & 2,4 & 4 & 3 & 1,8 & 2,8 \\
\hline Vital Tones Depression Pro & 2,8 & 3 & 2,33 & 2,8 & 2,73 \\
\hline $\begin{array}{l}\text { Brainwaves - For sleep, relaxation \& } \\
\text { mental health }\end{array}$ & 2,6 & 3 & 3 & 2,2 & 2,7 \\
\hline Depression überwinden & 2,2 & 3 & 2,67 & 2,8 & 2,67 \\
\hline Meeresrauschen & 1,8 & 3,75 & 2,33 & 2,67 & 2,64 \\
\hline Depressionen Pocket $^{c}$ & 2,6 & 2,5 & 3 & 2,4 & 2,63 \\
\hline Depressionen Pocket $^{c}$ & 1,8 & 3 & 2,67 & 2 & 2,37 \\
\hline Our Lady of Knots Undoer & 2,2 & 2,75 & 2 & 2,4 & 2,34 \\
\hline Physiokomp, KHL Psychiatrie & 2 & 2,5 & 2,67 & 2 & 2,29 \\
\hline Vital tones Depression & 2,2 & 2,5 & 2 & 2,4 & 2,28 \\
\hline Psycho Walkman & 2 & 2,75 & 2 & 2,2 & 2,24 \\
\hline Test: Sind Sie depressiv? & 1,8 & 2,5 & 2 & 2 & 2,08 \\
\hline$M=$ & 2,88 & 3,11 & 3,03 & 3,01 & 3,01 \\
\hline
\end{tabular}

bei $\mathrm{M}=3,12(\mathrm{SD}=0,48)$, in der Subdimension «Ästhetik» bei $\mathrm{M}=$ $3,01(\mathrm{SD}=0,62)$, in der Subdimension «Informationsqualität» bei $\mathrm{M}=2,98$ ( $\mathrm{SD}=0,61)$ und bei der «Nutzereinbindung» betrug der Mittelwert $\mathrm{M}=2,89$ ( $\mathrm{SD}=0,64)$. Tabelle 1 zeigt die Mittelwerte der eingeschlossenen Apps für alle Dimensionen.
Die ICC der beiden Gutachter beim MARS-Rating war mit 0,944 (95\%-CI 0,936 bis 0,950) exzellent.

Zwischen dem Nutzerrating und den Mittelwerten der Dimensionen oder dem Gesamtmittelwert zeigten sich keine signifikanten bivariaten Korrelationen ( $p>0,05$; die vollständige Korrelationsta- 
Tab. 2. Theoretische Hintergründe und Inhalte von iTunes-Apps

\begin{tabular}{|c|c|c|c|c|c|c|c|c|c|}
\hline \multirow[t]{2}{*}{ Name } & \multicolumn{5}{|c|}{ Interventionselemente } & \multirow{2}{*}{$\begin{array}{l}\text { Information/ } \\
\text { Psychoedukation }\end{array}$} & \multirow[t]{2}{*}{ Assessment } & \multirow{2}{*}{$\begin{array}{l}\text { Monitoring/ } \\
\text { Tracking }\end{array}$} & \multirow[t]{2}{*}{ Andere } \\
\hline & $\begin{array}{l}\text { alternativ- } \\
\text { medizinisch }\end{array}$ & KVT & VT & $\begin{array}{l}\text { Dritte } \\
\text { Welle }\end{array}$ & andere & & & & \\
\hline \multicolumn{10}{|l|}{$\begin{array}{l}\text { Über MARS-Cut-off } \\
\text { Keine }\end{array}$} \\
\hline \multicolumn{10}{|l|}{ Unter MARS-Cut-off } \\
\hline $\begin{array}{l}\text { Brainwaves - For sleep, } \\
\text { relaxation \& mental } \\
\text { health }\end{array}$ & - & - & - & - & $\checkmark$ & - & - & - & - \\
\hline Depressed? & - & - & - & - & - & - & $\checkmark$ & - & - \\
\hline $\begin{array}{l}\text { Depression Test - Am I } \\
\text { Depressed Online } \\
\text { Personality Test App }\end{array}$ & - & - & - & - & - & - & $\checkmark$ & - & - \\
\hline $\begin{array}{l}\text { depression-be positive } \\
\text { mentality }\end{array}$ & - & - & - & - & - & - & $\checkmark$ & - & - \\
\hline Depressionen Pocket & - & - & - & - & - & $\checkmark$ & - & - & - \\
\hline $\begin{array}{l}\text { Get Rid Of Depression } \\
\text { With Chinese Massage } \\
\text { Points - FREE Acupressure } \\
\text { Treatment Trainer }\end{array}$ & $\checkmark$ & - & - & - & - & - & - & - & - \\
\hline $\begin{array}{l}\text { Get Rid Of DepressionWith } \\
\text { Chinese Massage Points - } \\
\text { PREMIUM Acupressure } \\
\text { Treatment Trainer }\end{array}$ & $\checkmark$ & - & - & - & - & - & - & - & - \\
\hline MoodLog & - & - & - & - & - & - & - & $\checkmark$ & - \\
\hline Vital Tones Depression & - & - & - & - & $\checkmark$ & - & - & - & - \\
\hline Vital Tones Depression Pro & - & - & - & - & $\checkmark$ & - & - & - & - \\
\hline $\mathrm{n}=$ & 2 & 0 & 0 & 0 & 3 & 1 & 3 & 1 & 0 \\
\hline
\end{tabular}

VT $=$ Verhaltenstherapie; KVT $=$ kognitive Verhaltenstherapie; MARS = Mobile Application Rating Scale.

belle kann bei den Autoren angefragt werden). Eine Post-hoc-Poweranalyse ergab eine Power von $1-\beta=0,86\left(\alpha=0,05 ; r_{\mathrm{H} 1}=0,55\right.$ (vgl. Hides et al. [2014]; $\mathrm{n}=26$ ).

\section{Inhalte und theoretische Hintergründe}

Tabelle 2 (iTunes) und Tabelle 3 (Android) zeigen systematisch die Inhalte der Apps. 13 Apps (34\%) beinhalteten ein Assessment, 7 Apps (18\%) Informationen über Depression bzw. psychoedukative Inhalte, 6 Apps (16\%) Monitoring- und Tracking-Funktionen. Hinsichtlich Interventionselementen waren alternativmedizinische Elemente (z.B. Akupressur) bei 6 Apps (16\%), Elemente der dritten Welle der VT bei 4 Apps (11\%) und Elemente der KVT und VT bei je 2 Apps (5\%) zu finden. Sieben Apps (18\%) enthielten andere Interventionselemente (z.B. Hypnose). Die Inhalte von insgesamt 6 Apps (z.B. Bibelverse) fielen in die Kategorie «andere».

Die Assessment-Inhalte der Apps reichten von nichtwissenschaftlichen Fragen (z.B. «Mögen Sie schwarze Kleidung?») bis hin zu wissenschaftlich geprüften Fragebögen (z.B. PHQ-9, MyM3 oder Fragen aus dem SKID-IV). Bei 7 Apps wurde am Ende eine Diagnose gestellt (z.B. «mittelschwere Depression»). Die Diagnosen erfolgten anhand der dimensionalen Erfassung der depressiven Symptomatik. In den meisten Fällen wurde darauf hingewiesen, dass diese Diagnose kein Arztgespräch ersetzt und dass ein Arzt oder Therapeut aufgesucht werden sollte. Bei 2 Apps gab es diesen Hinweis jedoch nicht. Zwei Apps verwiesen auf externe Hilfeseiten, wenn ihr Assessment einen Hinweis auf eine erhöhte Suizidgefahr ergab.

Alle Apps mit psychoedukativen Inhalten gingen auf den $\mathrm{Be}$ griff «Depression» ein, 5 Apps zudem auf den Verlauf und die Prognose, 4 Apps auf die deskriptive Epidemiologie und 3 Apps auf die Ätiopathogenese.

\section{Praxis- und therapierelevante Apps}

Vier Apps überschritten die Cut-off-Werte von 3,5 beim Gesamtrating und 4,0 in der Subdimension «Informationsqualität». Die Apps «Back to Job», «Enke-App», «Stimmungstagebuch BETA» sowie «Emotion» werden deshalb in Bezug auf ihren klinischen Nutzen im Folgenden näher beschrieben.

Back to Job

- App-Name: Back to Job;

- Anbieter: Jambo9;

- Link: https://play.google.com/store/apps/details?id=de.lundbeck. backtojob\&hl=de;

- Funktionen: Tagebuchfunktion für depressive Symptome (Export möglich), Tipps gegen Depression. 
Tab. 3. Theoretische Hintergründe und Inhalte von Android-Apps

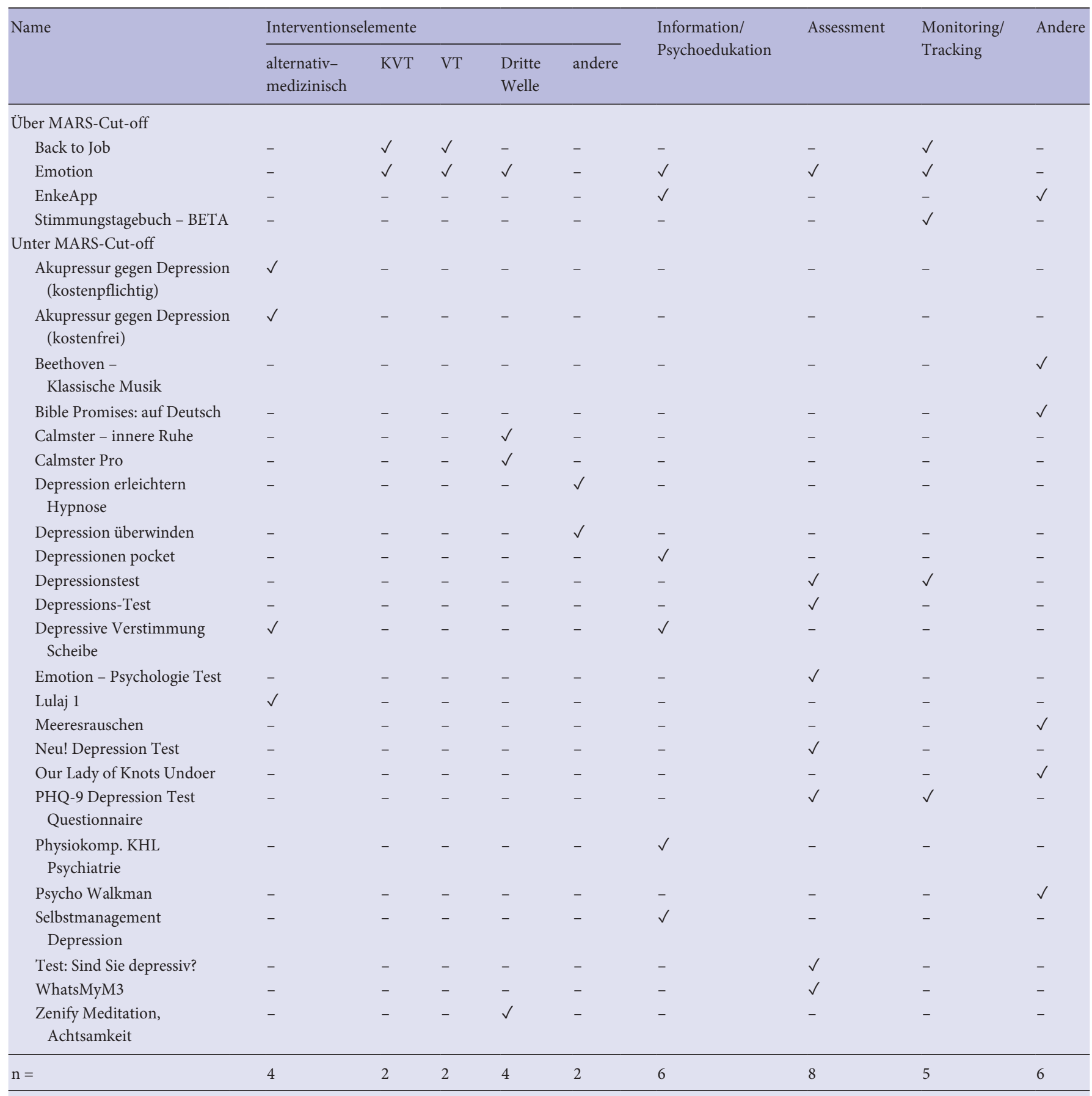

VT = Verhaltenstherapie; KVT = kognitive Verhaltenstherapie; MARS = Mobile Application Rating Scale.

Beurteilung: Die von der Brand Health GmbH im Auftrag der Pharmafirma Lundbeck GmbH entwickelte App «Back to Job» bietet eine intuitive Tagebuchfunktion mit übersichtlicher Auswertungsmöglichkeit für depressionsspezifische Symptome. Obwohl der Name und die Beschreibung eine Verbindung mit einer Rückkehr zum Arbeitsplatz (z.B. nach stationärem Aufenthalt) vermuten lassen, ist die App weitestgehend unabhängig davon nutzbar. Besonders viel Wert legen die Entwickler auf Datenschutz; Auswertungen können einfach per E-Mail mit z.B. dem behandelnden
Psychotherapeuten oder Arzt geteilt werden. Eine Reihe von «Tipps für den Alltag» erscheinen aus klinischer Perspektive für eine allgemeine Beratung bei Depression angemessen.

«Back to Job» enthält eine ausführliche Erklärung zum Datenschutz. Es werden keine personenbezogenen Informationen gespeichert. Eine mögliche Risikoquelle stellt die E-Mail-Funktion dar, mit der sich Kalendereinträge exportieren lassen. Jedoch wird explizit darauf hingewiesen, dass die Verantwortung für die Sicherheit des E-Mail-Kontos beim Benutzer liegt. 
Stimmungstagebuch - BETA

- App-Name: Stimmungstagebuch - BETA;

- Anbieter: Jonathan Sautter;

- Link: https://play.google.com/store/apps/details?id=de. jonathansautter.stimmungstagebuch\&hl=de.

Beurteilung: Die einfach zu handhabende App bietet die Möglichkeit des Stimmungs-Monitoring, und diverse andere Parameter, die individuell ergänzt und ausgewählt werden können, lassen sich aufzeichnen. Die Hersteller verzichten darauf, weitere therapeutische Inhalte vermitteln zu wollen. Die App bietet einen Passwortschutz, eine Erinnerungs-, Sende- und Speicherfunktion. Eine übersichtliche Auswertung kann so an den behandelnden Psychiater oder Psychotherapeuten übermittelt oder ausgedruckt werden. Aus klinischer Sicht ist dieses Programm bei diversen Erkrankungen als Tagebuch-App für Patienten geeignet, die eine digitale Version der herkömmlichen Tagebücher bevorzugen.

«Stimmungstagebuch - BETA» enthält keine Informationen zum Datenschutz. Der Zugriff auf die App kann aber zumindest lokal durch ein Passwort, das beim Öffnen der App eingegeben werden muss, geschützt werden. Auf Risiken für die Datensicherheit, die durch die vorhandene Exportfunktion entstehen, wird nicht verwiesen.

\section{Emotion}

- App-Name: Emotion;

- Anbieter: LES LABORATOIRES SERVIER;

- Link: https://play.google.com/store/apps/details?id=com.servier. emotione hl=de.

Beurteilung: Die nach unserem Download ausschließlich auf dem Tablet nutzbare und von dem Pharmahersteller Servier produzierte App «Emotion» bietet ein depressionsspezifisches Monitoring anhand eines nicht näher bezeichneten Fragebogens sowie Informationen zum Krankheitsbild, «Tipps» zur Lebensführung, Erfahrungsberichte sowie Entspannungstechniken als Videos an. Eine Erinnerungs- und Sendefunktion ist ebenfalls enthalten. Die Informationen zu Depression sind teilweise inhaltlich-wissenschaftlich diskutierbar, nähere Informationen zu dem zugrundeliegenden Fragebogen fehlen. Das Monitoring ist nicht individuell anpassbar, und die Patientenberichte lesen sich unzusammenhängend.

Die «Emotion»-Applikation garantiert die vertrauliche Behandlung der Daten. Die Rechte auf das Teilen der Daten liegen beim Nutzer. Die genaue Art der Vertraulichkeit oder die technische Umsetzung werden nicht beschrieben und sind daher fragwürdig.

\section{EnkeApp}

- App-Name: EnkeApp;

- Anbieter: Robert-Enke-Stiftung;

- Link: https://play.google.com/store/apps/details?id=de.robert enkestiftung.apperhl=de.

Beurteilung: Die übersichtlich gestaltete und von der Robert-

Enke-Stiftung mit seinen Partnern mobfish GmbH und migardo $\mathrm{GmbH}$ entwickelte «EnkeApp» bietet fundierte Informationen zu Depressionen und Suizidalität, einen Depressionstest sowie einen
Mood-Tracker. Das Kernelement ist der SOS-Bereich. Hier kann entweder eine kostenfreie Beratungshotline der Universitätsklinik Aachen angerufen oder ein Hilferuf an ein persönliches FreundeNetzwerk abgesetzt werden. Eine durchgängig erreichbare Notfallzentrale kann kostenpflichtig ergänzt werden. Aus klinischer Perspektive erscheinen die Informationen zu Depressionen übersichtlich und inhaltlich korrekt aufgearbeitet. Während der Social-Media-Bereich der App für Patienten eher ungeeignet erscheint, könnte die SOS-Funktion für Patienten mit latenter Suizidalität hilfreich sein.

Eine Erklärung zum Datenschutz ist im Impressum der EnkeApp angeführt. Grundsätzlich werden hier keine personenbezogenen Daten erhoben. Der Inhalt der App ist psychoedukativ, und es wird auf externe Seiten verlinkt. Für die Datenhandhabung auf den Seiten übernimmt die App keine Haftung.

\section{Diskussion}

\section{Zentrale Ergebnisse}

Dies ist die erste Studie zur systematischen Untersuchung der Charakteristika, Qualität und Inhalten von Apps in deutschen App-Stores, die ausdrücklich mit der Thematik «Depression» oder «Depressivität» werben. Die allgemeine Qualität der eingeschlossenen Apps war mittelmäßig $(\mathrm{M}=3,02 ; \mathrm{SD}=0,51)$. Lediglich 4 der 38 untersuchten Apps überschritten die zuvor definierten Cut-offWerte im Gesamtrating und in der Informationsqualität. Für keine der eingeschlossenen Apps konnte eine wissenschaftliche Studie zu deren Wirksamkeit/Nutzen für die klinische Praxis gefunden werden.

Der festgestellte Evidenzmangel deckt sich mit den Ergebnissen von Übersichtsarbeiten [Donker et al., 2013] und der Studie von Bardus et al. [2016], die Apps zum Gewichtsmanagement mit der MARS evaluierten. Auch wenn viele angebotene Apps eher den Charakter von Therapiebegleitmaterial (z. B. Psychoedukation) haben, sollten wissenschaftliche Studien mögliche Effekte, Risiken und Nebenwirkungen von solchen Apps untersuchen, die mit Bezug auf psychischen Störungen eingesetzt werden sollen bzw. mit dem Fokus auf Depression angeboten werden.

Eine mögliche Art der Anwendung ist das ambulante Depressions-Assessment, eine Funktion, die 34\% der eingeschlossenen Apps beinhalteten. Bei 7 Apps wurde, basierend auf dem Ergebnis der dimensionalen Erfassung der depressiven Symptomatik, ohne klassifikatorische und differenzialdiagnostische Abwägungen und ohne den Einbezug eines behandelnden Psychotherapeuten eine Diagnose gestellt. Die Bundesärztekammer, die Bundespsychotherapeutenkammer sowie die Kassenärztliche Bundesvereinigung weisen darauf hin, dass die «Diagnosestellung in Deutschland aufgrund ihrer Schwierigkeit, ihrer Gefährlichkeit und Unvorhersehbarkeit etwaiger Reaktionen der Patienten in den Bereich der höchstpersönlichen Leistungen des Psychotherapeuten oder Arztes fällt» [Bundesärztekammer und Kassenärztliche Bundesvereinigung, 2008; Bundespsychotherapeutenkammer, 2017]. Aus klinischer Sicht ist ein derartiges Vorgehen unzulässig, insbesondere 
wenn kein Hinweis gegeben wird, dass für eine Diagnose ein Besuch bei einem Arzt oder Psychotherapeuten unerlässlich ist (dies traf auf 2 der eingeschlossenen Apps zu). Keine der eingeschlossenen Apps verfügte über ausreichende Elemente, um mögliche Reaktionen von Nutzern zu erkennen oder aufzufangen. Allerdings verwiesen 2 der Assessment-Apps auf Hilfestellen, falls suizidale Gedanken angegeben wurden.

Neben ambulanten Assessments wiesen die Apps auch psychoedukative Inhalte (18\%), Monitoring- und Tracking-Funktionen (16\%) und verschiedene Interventionselemente auf. Die Informationsqualität der App-Inhalte wurde als mittelmäßig $(\mathrm{M}=2,98$; $\mathrm{SD}=0,61$ ) beurteilt. Den enthaltenen Informationen mangelte es häufig sowohl an Korrektheit als auch an qualitativer Aufbereitung und Präsentation. Aus klinischer Sicht ist insbesondere die Verbreitung von Fehlinformationen zur Ätiopathogenese und Behandlung von Depression äußerst alarmierend.

Im Einklang mit Bardus et al. [2016] konnten keine signifikanten Korrelationen zwischen dem Nutzerrating in den App-Stores und der Informationsqualität oder dem Gesamtrating ermittelt werden. Somit eignet sich das Nutzerrating weder für eine hinreichende Einschätzung der Informationsqualität noch der Gesamtqualität der Apps [Bardus et al., 2016; Armstrong, 2015; Nicholas et al., 2015].

Für Betroffene und Behandler wären zentrale Bewertungseinrichtungen zur wissenschaftlichen und qualitativen Überprüfung und Kategorisierung von Apps sehr nützlich. Auf diese Weise könnten Anwender schnell passende Apps finden. Kriterien für ein solches Gütesiegel sollten unter anderem 1) die Richtigkeit und wissenschaftliche Prüfung der App-Inhalte, 2) eine hohe Qualität (z.B. MARS-Rating), 3) gestufte Sicherheitsvorkehrungen für Krisensituationen (z.B. Suizidalität) und 4) ein ausreichender Datenschutz sein. In dieser Studie wiesen lediglich 2 Apps einen lokalen Passwortschutz auf. Darüber hinaus könnten bestehende Kriterien zur Beurteilung des Einsatzes internetbasierter Interventionen (z.B. die Integrierbarkeit in die Versorgung oder gesundheitsöknomische Aspekte) [Klein et al., 2016] für Apps adaptiert und bei Gütesiegel angewandt werden. Während die technische Sicherheit von Gesundheits-Apps künftig durch das Medizinproduktegesetz (für weitere Informationen zum Medizinproduktegesetz siehe unter anderem Pramann [2016a]) geregelt wird, gibt es für die inhaltliche Qualität keine Regelungen. Ein Gütesiegel, das diese berücksichtigt, wäre eine wertvolle Ergänzung.

Um das aktuelle App-Angebot (z.B. für andere psychische Störungen) zu evaluieren und transparenter zu machen, sind weitere Übersichtsarbeiten nötig. Bei systematischen Übersichtsarbeiten sollte darauf geachtet werden, dass die Suche nicht auf bestimmte Kategorien begrenzt wird [vgl. Bardus et al., 2016] und nicht nur populäre Apps eingeschlossen werden [vgl. Bardus et al., 2016; Nicholas et al., 2015; Armstrong, 2015]. Künftige Forschung sollte zudem die Effektivität und Güte von bestehenden Apps überprüfen und neue, effektive und evidenzbasierte Apps entwickeln und in die Praxis übertragen oder bereits in anderen Sprachen vorliegende Apps übersetzen. Dabei sollte auch ein besonderer Fokus auf die Informationssicherheit und den Datenschutz gelegt wer- den, da dies für Therapeuten und App-Nutzer besonders wichtig ist. Der Datenschutz beschreibt das Recht jeder Person auf informationelle Selbstbestimmung und hat zum Ziel, vor der missbräuchlichen Verwendung von Daten zu schützen. In Deutschland wird der Datenschutz über das Bundesdatenschutzgesetz (BDSG) geregelt und befasst sich ausschließlich mit personenbezogenen Daten. Zudem tritt am 25. Mai 2018 Die EU-Datenschutz-Grundverordnung (EU-DSGVO) in Kraft. EU-Verordnungen sind allgemeine Regelungen mit unmittelbarer innerstaatlicher Geltung. Die EU-DSGVO regelt den Schutz der Grundrechte und Grundfreiheiten von Personen in Bezug auf personenbezogene Daten und deren freien Verkehr. Sie stellt ein Verbotsgesetz mit Erlaubnisvorbehalt dar. Das bedeutet, dass mit personenbezogenen Daten nicht umgegangen werden darf, außer die Person willigt freiwillig und informiert selbst ein oder eine gesetzliche Vorschrift erlaubt dies (für weitere Informationen zum Datenschutz im Rahmen von Gesundheits-Apps siehe unter anderem Pramann [2016b]). Der Datenschutz hat für Gesetzgeber Leistungserbringer und Nutzer gleichermaßen einen hohen Stellenwert. Seitens der Europäischen Kommission [European Commission, 2014, 2016] gibt es Bemühungen, Richtlinien zur Erfassung und zum Umgang mit personenbezogenen Daten durch «mHealth»-Applikationen zu erstellen. Die Einhaltung dieser angestrebten Richtlinien wäre derzeit nicht gewährleistet [Ruck et al., 2016]. Allerdings lieferten Ruck et al. [2016] Leitlinien für die Entwicklung künftiger Gesundheits-Apps. Insgesamt stellt der Mangel an globalen Sicherheitsstandards sowie an Standards zur inhaltlichen Güte von Apps eine bedeutende Barriere für die Nutzung von «mHealth»-Applikationen dar.

Für die Praxis zeigte sich, dass es bereits eine Vielzahl von Apps gab, die explizit mit der Thematik «Depression/Depressivität» werben und unterschiedliche Inhalte von Informationen bis hin $\mathrm{zu}$ Interventionen anbieten. Diese Studie konnte unter Berücksichtigung der unten genannten Limitationen zeigen, dass die allgemeine Qualität der beurteilten Apps mittelmäßig war und es für Nutzer schwierig war, dies zu erkennen. Auch waren die Inhalte der Apps nicht wissenschaftlich geprüft. In ihrer jetzigen Form stellen die App-Stores und Apps nur eine stark eingeschränkte Hilfe für von Depression Betroffene dar, und die Leistungsbringer sollten ihre Nutzer darauf hinweisen, dass 1) die technische Sicherheit der Apps nicht geprüft wurde, 2) der Datenschutz nicht vollständig gegeben ist und 3) die Wirksamkeit der Applikation noch nicht in randomisiert-kontrollierten klinischen Studien bestätigt wurde. Das gilt auch für die 4 hier als gegebenenfalls praxis- und therapierelevant eingestuften Apps.

\section{Limitationen}

Aufgrund der hohen Frequenz der Neu- und Weiterentwicklungen von Apps [Albrecht, 2016a] ist es möglich, dass sich Inhalte bei der hier eingeschlossenen Apps inzwischen verändert haben und das Rating somit bei einer erneuten Evaluation anders ausfällt oder dass neue Apps eingeschlossen werden könnten bzw. in die vorliegende Untersuchung eingeschlossene Apps nicht mehr zur Verfügung stehen. 
Die oben ausgesprochenen Empfehlungen für den Einsatz der 4 über den Cut-off-Werten liegenden Apps basieren nicht auf wissenschaftlichen Studien, sondern beruhen auf einem Expertenurteil. Die verwendeten Cut-off-Werte zum Qualitätsrating ergaben sich auf der Basis theoretischer Überlegungen und unter Berücksichtigung der inhaltlichen Bedeutung der Skalenmetrik ( 4 = gute Informationsqualität; 3,5 = überdurchschnittliche Gesamtqualität). Das Qualitätsrating konnte dabei objektiv und reliabel mit dem multidimensionalen MARS-Rating durchgeführt werden (ICC = 0,944). Somit konnte die Qualität der Apps in den Dimensionen «Nutzereinbindung», «Funktionalität», «Ästhetik» und «Informationsqualität» verlässlich eingeschätzt werden. Durch die Anpassungen der Klassifikationsseite konnten zudem Inhalte nach theoretischen Hintergründen differenziert werden.

Die MARS ist jedoch nicht in der Lage, psychotherapeutische Qualitätsmerkmale quantitativ zu erfassen, was andere Messinstrumente wie das englischsprachige «Enlight»-Tool bieten [Baumel et al., 2017]. In der fortlaufenden deutschen Übersetzung (www.researchgate.net/project/German-version-of-the-Mobile-App-RatingScale-MARS-G) der MARS wird derzeit eine Skala zur psychotherapeutischen Qualität ergänzt. Die methodische Überprüfung sowohl der MARS als auch des «Enlight»-Tools weisen Mängel auf. So beruhen die Skalenanalysen bei beiden Instrumenten primär auf Cronbachs-Alpha-Werten, was kritisch diskutiert wird [Dunn et al., 2014; Revelle und Zinbarg, 2009; McDonald, 1999], und beide überprüfen das angenommene Messmodell nicht auf latenter Ebene. Die Cronbachs-Alpha-Werte und IRR beider Instrumente entsprechen häufig verwendeten Maßstäben [Fleiss, 1999; Baumel et al., 2017; Stoyanov et al., 2015; George und Mallery, 2003]. Bei der hier durchgeführten Analyse ließen sich im Gegensatz zu den Befunden im Validierungsprozess des MARS-Ratings keine signifikanten Korrelationen zwischen MARS-Rating und Nutzerrating feststellen [Hides et al., 2014]. Künftige Studien im deutschsprachigen Raum sollten in Betracht ziehen, die deutschsprachige Version der MARS nach deren Validierung zu verwenden, um potenzielle Effekte durch die Sprache zu vermeiden und die psychotherapeutische Qualität quantitativ erfassen zu können. Für Letzteres stellt das «Enlight»-Tool [Baumel et al., 2017] eine englischsprachige Alternative dar.

Der in dieser Studie angewandte Suchstring wurde entlang der depressiven Störung bzw. Depressivität entwickelt. So konnten Apps identifiziert werden, die explizit für Depression/Depressivität entwickelt und verschlagwortet wurden und in den Appstores verfügbar waren. Entsprechend wurde die Qualität von Apps bewertet, die von potenziellen in App-Stores explizit nach «Depression» suchenden Nutzer gefunden werden konnten. Diese Übersichtsarbeit zielte somit auf die Qualität von Apps, die Tools/Interventionen/Behandlungen im Störungskontext bewarben und somit einen erhöhten Qualitätsstandard erfüllen sollten. Apps, die potenziell ebenfalls zur Verbesserung depressiver Stimmung hätten beitragen können, aber nicht explizit mit der Thematik «Depression/ Depressivität» warben, wie z.B. Apps für körperliche Aktivität, Schlafhygiene-Apps und Stressfrei-Apps, wurden nicht berücksichtigt. Apps, die zur Behandlung von Depression relevante Me- thoden (z.B. Modifikation kognitiver Verzerrungen) anboten oder mit mit Depression in Verbindung stehenden Begriffen (z.B. Lebensqualität oder Resilienz) warben, aber nicht explizit mit Depression bzw. Depressivität verschlagwortet wurden, gingen ebenfalls nicht in die Analyse ein.

Mit dem Einschluss solcher Apps, von Apps aus Forschungsprojekten, die nicht über die App-Stores bezogen werden können, oder auch von Apps, die auf bestimmte Symptome von Depression (z.B. Antriebslosigkeit) abzielen sowie mit der Betrachtung von anderen Störungen könnten künftige Studien eine wertvolle Ergänzung zu dieser Arbeit leisten und mehr Transparenz und Qualität in diesem bisher kaum kontrollierten Gesundheitsmarkt schaffen.

Grundsätzlich könnte das Erstellen von systematischen Übersichtsarbeiten zu Apps künftig durch die Verwendung sogenannter Webcrawler-Programme, die das Internet und App-Stores systematisch nach Apps durchsuchen und bestimmte Informationen auslesen, deutlich vereinfacht werden. Idealerweise könnten so Datenbanken aufgebaut werden, die wichtige Informationen zu Apps speichern und ähnliche Funktionen wie Literaturdatenbanken übernehmen. Forscher wären so nicht mehr auf eine Suche in den jeweiligen Stores angewiesen und müssten Informationen von Apps nicht einzeln aus den Store-Seiten extrahieren. Solche Datenbanken existieren bereits für einige Länder (USA, Russland, Japan, China, Brasilien) [Xu und Liu, 2015], fehlen aber für den deutschsprachigen Raum bzw. befinden sich noch in der Entwicklungsphase (z.B. www.mhad.science).

\section{Zusammenfassung}

Dies ist die erste systematische Übersichtsarbeit zu deutschsprachigen Apps in deutschen App-Stores, die explizit unter «Depression» verschlagwortet sind. Zwar ließ sich eine Vielzahl von Apps finden $(\mathrm{N}=1156)$, jedoch konnte nur ein sehr kleiner Anteil als relevant für die klinische Praxis beurteilt werden $(n=4)$. Für keine der eingeschlossenen Apps konnten wissenschaftliche Studien zu Wirksamkeit, Nutzen und Risiken gefunden werden.

Von Depression Betroffene wie auch Leistungserbringer können daher große Schwierigkeiten haben, eine qualitativ hochwertige App mit evidenzbasierten Inhalten zu finden. Systematische Übersichtsarbeiten wie diese sind daher von zentraler Bedeutung, um auf der Basis wissenschaftlicher Methoden über die Qualität von Apps zu informieren. Zur Beurteilung der Qualität von Apps sowie der Daten- und Patientensicherheit könnte ein Gütesiegel helfen. Auf diese Weise könnten relevante und empfehlenswerte Apps leichter identifiziert und von Depressionen betroffene Menschen vor Missbrauch und Fehlinformationen geschützt werden.

\section{Disclosure Statement}

Die Autoren erklären, dass keine Interessenskonflikte vorliegen. Alle Kosten wurden durch das Budget der Abteilung für Klinische Psychologie und Psychotherapie, Institut für Psychologie und Pädagogik, Universität Ulm, gedeckt. 


\section{Literatur}

Aitken M: Patient Adoption of mHealth: Use, Evidence and Remaining Barriers to Mainstream Acceptance. Parsippany, NJ, IMS Institute for Healthcare Informatics, 2015.

Albrecht U-V (ed): Chancen und Risiken von Gesundheits-Apps (CHARISMHA). Hannover, Medizinische Hochschule Hannover, 2016a.

Albrecht U-V: Kapitel 8. Gesundheits-Apps und Risiken; in Albrecht U-V (ed): Chancen und Risiken von Gesundheits-Apps (CHARISMHA). Hannover, Medizinische Hochschule Hannover, 2016b, pp 176-192.

Andrade LH, Alonso J, Mneimneh Z, et al: Barriers to mental health treatment: results from the WHO World Mental Health surveys. Psychol Med 2014;44:13031317.

Armstrong S: Which app should I use? BMJ 2015;351: h4597.

Bakker D, Kazantzis N, Rickwood D, Rickard N: Mental health smartphone apps: review and evidence-based recommendations for future developments. JMIR Ment Health 2016;3:e7.

Bardus M, van Beurden SB, Smith JR, Abraham C: A review and content analysis of engagement, functionality, aesthetics, information quality, and change techniques in the most popular commercial apps for weight management. Int J Behav Nutr Phys Act 2016;13:35.

Baumel A, Faber K, Mathur N, et al: Enlight: a comprehensive quality and therapeutic potential evaluation tool for mobile and web-based eHealth interventions. J Med Internet Res 2017;19:e82.

Bundesärztekammer und Kassenärztliche Bundesvereinigung: Persönliche Leistungserbringung: Möglichkeiten und Grenzen der Delegation ärztlicher Leistungen. www.bundesaerztekammer.de/richtlinien/empfehlungenstellungnahmen/delegation/, 2008 (letzter Abruf 9. April 2018).

Bundespsychotherapeutenkammer: Internet in der Psychotherapie. www.bptk.de/fileadmin/user_upload/Publikationen/BPtK-Standpunkte/Internet_in_der_Psychotherapie/20170629_bptk_standpunkt_internet.pdf, 2017 (letzter Abruf 9. April 2018)

Burns MN, Begale M, Duffecy J, et al: Harnessing context sensing to develop a mobile intervention for depression. J Med Internet Res 2011;13:e55.

Carter MC, Burley VJ, Nykjaer C, Cade JE: Adherence to a smartphone application for weight loss compared to website and paper diary: pilot randomized controlled trial. J Med Internet Res 2013;15:e32.

Cerwall P, Jonsson P, Möller R, et al: ERICSSON Mobility Report MWC 2015: On the Pulse of the Networked Society. www.ericsson.com/res/docs/2015/ericssonmobility-report-feb-2015-interim.pdf, 2015 (letzter Abruf 9. April 2018).

Charani E, Castro-Sánchez E, Moore LSP, Holmes A: Do smartphone applications in healthcare require a governance and legal framework? It depends on the application! BMC Med 2014;12:29.

DGPPN, BÄK, KBV, et al: S3-Leitlinie/Nationale VersorgungsLeitlinie Unipolare Depression: Langfassung, 2015. www.leitlinien.de/mdb/downloads/nvl/depression/depression-2aufl-vers5-lang.pdf (letzter Abruf 9. April 2018)

Dicianno BE, Parmanto B, Fairman AD, et al: Perspectives on the evolution of mobile (mHealth) technologies and application to rehabilitation. Phys Ther 2015;95:397405.

Donker T, Petrie K, Proudfoot J, et al: Smartphones for smarter delivery of mental health programs: a systematic review. J Med Internet Res 2013;15:e247.

Dunn TJ, Baguley T, Brunsden V: From alpha to omega: a practical solution to the pervasive problem of internal consistency estimation. Br J Psychol 2014;105:399-412.
Endl R, Jäschke T, Thiel C, Wickinghoff DV: mHealth im Kontext des elektronischen Patientendossiers: Eine Studie im Auftrag von eHealth Suisse. St.Gallen, Fachhochschule St.Gallen, 2015. www.e-health-suisse.ch/ fileadmin/user_upload/Dokumente/2015/D/150309_ Bericht_FHSG_mHealth_D.pdf (letzter Abruf 9. April 2018).

European Commission: Green Paper on mobile Health («mHealth»). Brussels, 2014.

European Commission: Draft Code of Conduct on privacy for mobile health applications, 2016.

Ferrari AJ, Charlson FJ, Norman RE, et al: Burden of depressive disorders by country, sex, age, and year: findings from the global burden of disease study 2010. PLoS Med 2013a;10:e1001547.

Ferrari AJ, Somerville AJ, Baxter AJ, et al: Global variation in the prevalence and incidence of major depressive disorder: a systematic review of the epidemiological literature. Psychol Med 2013b;43:471-481.

Fleiss JL: The Design and Analysis of Clinical Experiments. Hoboken, NJ, John Wiley \& Sons, 1999.

George D, Mallery P: SPSS for Windows Step by Step: A Simple Guide and Reference: 11.0 Update, ed 4. Boston, Allyn \& Bacon, 2003.

Google: Unser mobiler Planet: Deutschland: Der mobile Nutzer. www.bvdw.org/mybvdw/media/download/ourmobile-planet-germany-de.pdf?file $=2266,2012$.

Gupta A, Sinha S, Schumacher J: Digital health: A way for pharma companies to be more relevant in healthcare. www.strategyand.pwc.com/reports/digital-health-pharma-companies, 2013 (letzter Abruf 9. April 2018).

Harrison V, Proudfoot J, Wee PP, et al: Mobile mental health: review of the emerging field and proof of concept study. J Ment Health 2011;20:509-524.

Hides L, Kavanagh D, Stoyanov SR, et al: Mobile Application Rating Scale (MARS): a new tool for assessing the quality of health mobile application, 2014. www.youngandwellcrc.org.au/wp-content/uploads/2015/03/ MARS-REPORT_FINAL_181214.pdf.

Jacobi F, Höfler M, Strehle J, et al: Mental disorders in the general population: study on the health of adults in Germany and the additional module mental health (DEGS1-MH). Nervenarzt 2014;85:77-87.

Kampling H, Baumeister H, Jäckel WH, Mittag O: Prevention of depression in chronically physically ill adults (protocol). Cochrane Database Syst Rev 2014; 8:CD011246.

Kauer SD, Reid SC, Crooke AHD, et al: Self-monitoring using mobile phones in the early stages of adolescent depression: randomized controlled trial. J Med Internet Res 2012;14:e67.

Klasnja P, Pratt W: Healthcare in the pocket: mapping the space of mobile-phone health interventions. J Biomed Inform 2012;45:184-198.

Klein JP, Gerlinger G, Knaevelsrud C, et al: Internetbasierte Interventionen in der Behandlung psychischer Störungen. Nervenarzt 2016;87:1185-1193.

Kuhn B, Amelung V: Kapitel 4. Gesundheits-Apps und besondere Herausforderungen; in Albrecht U-V (ed): Chancen und Risiken von Gesundheits-Apps (CHARISMHA). Hannover, Medizinische Hochschule Hannover, 2016, pp 100-114.

Liu C: Worldwide Internet and Mobile Users: eMarketer's Updated Estimates for 2015. https://insights.ap.org/ uploads/images/eMarketer_Estimates_2015.pdf, 2015 (letzter Abruf 9. April 2018).

Luxton DD, McCann RA, Bush NE, et al: mHealth for mental health: integrating smartphone technology in behavioral healthcare. Prof Psychol Res Pr 2011;42: 505-512.
Mack S, Jacobi F, Gerschler A, et al: Self-reported utilization of mental health services in the adult German population - evidence for unmet needs? Results of the DEGS1-Mental Health Module (DEGS1-MH). Int J Methods Psychiatr Res 2014;23:289-303.

McDonald RP: Test Theory: A Unified Treatment. Mahwah, NJ, Lawrence Erlbaum Associates Publishers, 1999.

Meeker M, Liang W: Internet Trends D11 Conference. http://kpcbweb2.s3.amazonaws.com/files/68/Internet Trends_052913_Final.pdf?1370374981, 2013 (letzter Abruf 9. April 2018).

Melchior H, Schulz H, Härter M: Faktencheck Gesundheit: Regionale Unterschiede in der Diagnositk und Behandlung von Depressionen. Gütersloh, Bertelsmann Stiftung, 2014. http://faktencheck-gesundheit.de/fileadmin/files/user_upload/Faktencheck_Depression_Studie. pdf (letzter Abruf 9. April 2018).

Nasi G, Cucciniello M, Guerrazzi C: The role of mobile technologies in health care processes: the case of cancer supportive care. J Med Internet Res 2015;17:e26.

Nicholas J, Larsen ME, Proudfoot J, Christensen H: Mobile apps for bipolar disorder: a systematic review of features and content quality. J Med Internet Res 2015;17:e198.

Pejovic V, Mehrotra A, Musolesi M: Anticipatory mobile digital health: towards personalised proactive therapies and prevention strategies. CoRR 2015;abs/1508.03722.

Pramann O: Gesundheits-Apps als Medizinprodukte; in Albrecht U-V (ed): Chancen und Risiken von Gesundheits-Apps (CHARISMHA). Hannover, Medizinische Hochschule Hannover, 2016a, pp 228-243.

Pramann O: Gesundheits-Apps und Datenschutz; in Albrecht U-V (ed): Chancen und Risiken von Gesundheits-Apps (CHARISMHA). Hannover, Medizinische Hochschule Hannover, 2016b, pp 214-227.

Prentice JL, Dobson KS: A review of the risks and benefits associated with mobile phone applications for psychological interventions. Can Psychol 2014;55:282-290.

Price M, Yuen EK, Goetter EM, et al: mHealth: a mechanism to deliver more accessible, more effective mental health care. Clin Psychol Psychother 2014;21:427-436.

Proudfoot J, Nicholas J: Monitoring and evaluation in low intensity CBT interventions; in Bennett-Levy J (ed): Oxford Guide to Low Intensity CBT Interventions. Oxford, Oxford University Press, 2010, pp 97-104

Proudfoot J, Parker G, Hadzi Pavlovic D, et al: Community attitudes to the appropriation of mobile phones for monitoring and managing depression, anxiety, and stress. J Med Internet Res 2010;12:e64

Reid SC, Kauer SD, Hearps SJC, et al: A mobile phone application for the assessment and management of youth mental health problems in primary care: a randomised controlled trial. BMC Fam Pract 2011;12:131.

Revelle W, Zinbarg RE: Coefficients alpha, beta, omega and the glb: Comments on Sijtsma. Psychometrika 2009;74:145-154.

Ruck A, Wagner Bondorf S, Lowe C: Second draft of guidelines: $\mathrm{EU}$ guidelines on assessment of the reliability of mobile health applications, 2016.

Rutz M, Kühn D, Dierks M-L: Kapitel 5. GesundheitsApps und Prävention; in Albrecht U-V (ed): Chancen und Risiken von Gesundheits-Apps (CHARISMHA). Hannover, Medizinische Hochschule Hannover, 2016a, pp 116-135.

Rutz M, Kühn D, Dierks M-L: Kapitel 6. GesundheitsApps und Diagnostik \& Therapie; in Albrecht U-V (ed): Chancen und Risiken von Gesundheits-Apps (CHARISMHA). Hannover, Medizinische Hochschule Hannover, 2016b, pp 136-159.

Shen N, Levitan M-J, Johnson A, et al: Finding a depression app: a review and content analysis of the depression app marketplace. JMIR Mhealth Uhealth 2015; 3:e16. 
Sobocki P, Jönsson B, Angst J, Rehnberg C: Cost of depression in Europe. J Ment Health Policy Econ 2006;9:8798.

Stoyanov SR, Hides L, Kavanagh DJ, et al: Mobile App Rating Scale: a new tool for assessing the quality of health mobile apps. JMIR Mhealth Uhealth 2015;3:e27.

Tomorrow Focus Media: Welche der folgenden Gesundheits-Apps nutzt Du? http://de.statista.com/statistik/ daten/studie/473432/umfrage/nutzung-von-gesundheits-apps-nach-themengebiet-und-geschlecht/, 2015.
Torous J, Powell AC: Current research and trends in the use of smartphone applications for mood disorders. Internet Interv 2015;2:169-173.

Warmerdam L, Riper H, Klein M, et al: Innovative ICT solutions to improve treatment outcomes for depression: the ICT4Depression project. Stud Health Technol Inform 2012;181:339-343.

Whittaker R, McRobbie H, Bullen C, et al: Mobile phonebased interventions for smoking cessation. Cochrane Database Syst Rev 2012;11:CD006611.
World Health Organization (WHO): Global burden of mental disorders and the need for a comprehensive, coordinated response from health and social sectors at the country level. http://apps.who.int/gb/ebwha/pdf files/EB130/B130_9-en.pdf, 2011 (letzter Abruf 9. April 2018)

Xu W, Liu Y: mHealthApps: a repository and database of mobile health apps. JMIR Mhealth Uhealth 2015;3:e28. 